East European Journal of Physics

PACS: $29.20 . \mathrm{db}, 29.27 . \mathrm{Bd}, 29.27 . \mathrm{Fh}$

\title{
INJECTION EFFICIENCY IN COMPTON RING NESTOR
}

\author{
P.I. Gladkikh, A.A. Kalamaiko, I.M. Karnaukhov, A.O. Mytsykov, A.Y. Zelinsky \\ National Scientific Center Kharkov Institute of Physics and Technology \\ 1, Akademicheskaya St., Kharkiv, 61108, Ukraine \\ E-mail:kalamaiko@kipt.kharkiv.ua \\ Received 5 August, 2017; accepted 1 October, 2017
}

NESTOR is the hard X-ray source that is under commissioning at NSC KIPT. NESTOR based on the Compton scattering of laser photons on relativistic electrons. The structure of the facility can be represented as the following components: a linear accelerator, a transport channel, a storage ring, and a laser-optical system. Electrons are stored in the storage ring for energy of 40-200 MeV. Inevitable alignment errors of magnetic elements are strongly effect on the beam dynamics in the storage ring. These errors lead to a shift of the equilibrium orbit relative to the ideal one. Significant shift of the equilibrium orbit could lead to loss of the beam on physical apertures. Transverse sizes of electron and laser beams are only few tens of microns at the interaction point. The shift of electron beam at the interaction point could greatly complicate the operation adjustment of storage ring without sufficient beam position diagnostic system. This article presents the simulation results of the efficiency of electron beam accumulation in the NESTOR storage ring. Also, this article is devoted to electron beam dynamics due to alignment errors of magnetic element in the ring.

KEYWORDS: Compton scattering, storage ring, alignment error, beam dynamics simulation, injection efficiency

\section{ЕФЕКТИВНІСТЬ ІНЖЕКЦІЇ ЕЛЕКТРОННОГО ПУЧКА В ГЕНЕРАТОР НЕСТОР \\ П.І. Гладких, А.А. Каламайко, І.М. Карнаухов, А.О. Мициков, А.Ю Зелінський Національний науковий цеентр «Харківський фізико-технічний інститут» 61108, Україна, м.Харків, вул Академічна, 1}

В ННЦ ХФТІ споруджується джерело жорсткого випромінювання «НЕСТОР», що базується на комптонівському розсіюванні лазерних фотонів на релятивістських електронах. Структурно джерело може бути представлено у вигляді наступних компонентів: лінійний прискорювач, канал транспортування, накопичувальне кільце, лазерно-оптична система. Електрони накопичуються в кільці на енергію 40-200 МеВ. Неминучі помилки юстирування магнітних елементів сильно впливають на динаміку електронного пучка в накопичувальному кільці, приводячи до зміщення положення рівноважної орбіти щодо ідеальної. Значні зміщення рівноважної орбіти можуть призводити до втрати пучка на фізичних апертурах накопичувача. Поперечні розміри електронного та лазерного пучків в точці зустрічі складають всього кілька десятків мікрон. Зміщення положення електронного пучка в точці взаємодії може сильно ускладнити настройку роботи джерела жорсткого випромінювання через фактичну відсутність належної системи індикації положення пучка. В даній статті наведені результати моделювання ефективності накопичення електронного пучка в накопичувачі НЕСТОР, а також розглядається вплив помилок юстирування магнітних елементів на його динаміку.

КЛЮЧОВІ СЛОВА: комптонівське розсіювання, накопичувальне кільце, помилки юстування, моделювання динаміки пучка, ефективність інжекції

\section{ЭФФЕКТИВНОСТЬ ИНЖЕКЦИИ ЭЛЕКТРОННОГО ПУЧКА В ГЕНЕРАТОР НЕСТОР \\ П.И. Гладких, А.А. Каламайко, И.М. Карнаухов, А.О. Мыциков, А.Ю. Зелинский \\ Национальный научный центр «Харьковский физико-технический институт»" 61108, Украина, г. Харьков, ул. Академическая, 1}

В ННЦ ХФТИ сооружается источник жесткого излучения «НЕСТОР», основанный на комптоновском рассеянии лазерных фотонов на релятивистских электронах. Структурно установка может быть представлена в виде следующих компонентов: линейный ускоритель, канал транспортировки, накопительное кольцо, лазерно-оптическая система. Электроны накапливаются в кольце на энергию 40-200 МэВ. Неизбежные ошибки юстировки магнитных элементов сильно влияют на динамику электронного пучка в накопительном кольце, приводя к смещению положения равновесной орбиты относительно идеальной. Значительные смещения равновесной орбиты могут приводить к потере пучка на физических апертурах накопителя. Поперечные размеры электронного и лазерного пучков в точке встречи составляют всего несколько десятков микрон. Смещение положения электронного пучка в точке взаимодействия может сильно осложнить настройку работы источника жесткого излучения из-за фактического отсутствия должной системы индикации положения пучка. В данной статье приведены результаты моделирования эффективности накопления электронного пучка в накопителе НЕСТОР, a также рассмотрено влияние ошибок юстировки магнитных элементов на его динамику.

КЛЮЧЕВЫЕ СЛОВА: комптоновское рассеяние, накопительное кольцо, ошибки юстировки, моделирование динамики пучка, эффективность инжекции

Идея генерации высокоэнергетичных фотонов на основе обратного комптоновского рассеяния была предложена в 1951 - 52 Х. Мотцем [1] и К. Ландекером [2]. После, метод обратного комптоновского рассеяния был развит в 1963 году Ф.Р. Арутюняном и В.А. Туманяном [3], а также независимо К.М. Мильбурном [4]. Но возможности ускорительной и лазерной техники в то время не позволяли рассматривать предложенный метод для практических целей из-за очень малого сечения этого процесса. 
В настоящее время, благодаря развитию ускорительной и лазерной техники открываются новые возможности в генерации жесткого излучения на основе обратного комптоновского рассеяния лазерных фотонов на релятивистских электронах. В мире уже существует несколько действующих источников жесткого излучения. Подобный источник сооружается и на Украине [5].

Цель данной работы заключается в оценке влияния ошибок юстировки магнитных элементов на динамику электронного пучка в накопителе «НЕСТОР».

\section{СТРУКТУРА ФОКУСИРОВКИ НАКОПИТЕЛЯ НЕСТОР}

Одна из основных проблем возникающих при проектировании подобной установки - получение приемлемой интенсивности генерируемого жесткого излучения из-за крайне малого значения сечения комптоновского рассеяния $\sigma_{\mathrm{c}} \sim 6,6 \times 10^{-29} \mathrm{M}^{2}$. При столкновении электронного и лазерного сгустков в накопителе интенсивность жесткого излучения определяется следующей формулой [6]

$$
\mathrm{I}_{\gamma}=\mathrm{L} \sigma_{\mathrm{c}} \mathrm{n}_{\mathrm{e}} \mathrm{n}_{\gamma} \mathrm{n}_{\mathrm{b}} \mathrm{f}_{\mathrm{rev}},
$$

где $\sigma_{\mathrm{c}}$-сечение комптоновского рассеяния, $\mathrm{n}_{\mathrm{e}}-$ количество электронов в одном сгустке, $\mathrm{n}_{\gamma}-$ количество лазерных фотонов в одном сгустке, $\mathrm{n}_{\mathrm{b}}$ - количество взаимодействующих электронных сгустков, $\mathrm{f}_{\text {rev }}-$ частота обращения электронного сгустка в накопителе, L-геометрическая функция, зависящая от поперечных и продольных размером электронного и лазерного сгустков, а также угла столкновения:

$$
\mathrm{L}=\frac{1}{2 \pi \sqrt{\left(\sigma_{\mathrm{ze}}^{2}+\sigma_{\mathrm{zl}}^{2}\right)} \sqrt{\left(\sigma_{\mathrm{xe}}^{2}+\sigma_{\mathrm{xl}}^{2}\right) \cos ^{2}(\varphi / 2)+\left(\sigma_{\mathrm{se}}^{2}+\sigma_{\mathrm{sl}}^{2}\right) \sin ^{2}(\varphi / 2)}},
$$

где $\sigma_{\mathrm{xe}}, \sigma_{\mathrm{xl}}, \sigma_{\mathrm{ze}}, \sigma_{\mathrm{zl}}$ - среднеквадратичные поперечные размеры в точке столкновения электронного и лазерного пучков, $\sigma_{\mathrm{se}}, \sigma_{\mathrm{sl}}$-среднеквадратичные продольные размеры электронного и лазерного сгустков, $\varphi-$ угол столкновения электронного и лазерного пучков. Поэтому, крайне важно обеспечить минимальные размеры электронного сгустка в точке взаимодействия.

Поперечные размеры электронного сгустка в накопителе определяются следующим выражением [7]

$$
\sigma_{x e, z e}=\sqrt{\varepsilon_{x, z} \beta_{x, z}+\left(D_{x, z} \delta\right)^{2}},
$$

где $\varepsilon_{x, z}-$ эмиттанс электронного пучка в горизонтальной (х) и вертикальной $(\mathrm{z})$ плоскости, $\beta_{x, z}-$ амплитудные функции в рассматриваемой точке, $\mathrm{D}_{\mathrm{x}, \mathrm{z}}-$ дисперсионная функция, $\delta=\Delta \mathrm{p} / \mathrm{p}-$ энергетический разброс в электронном сгустке. Как следует из формулы (3), чтобы получить малые поперечные размеры электронного сгустка в точке взаимодействия необходимо обеспечить минимальное значение амплитудных функций в этой точке, кроме того, промежуток взаимодействия должен быть бездисперсионным.

На рис. 1 приведена схема накопительного кольца «НЕСТОР» [8].

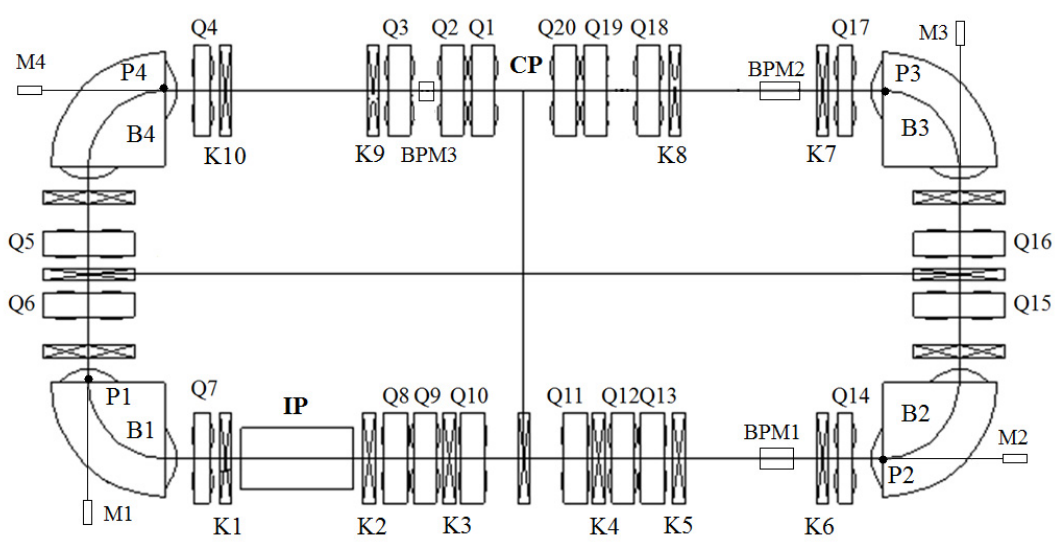

Рис.1. Схема накопительного кольца НЕСТОР

В1 - В4 - поворотные магниты, Q1 - Q20 - квадрупольные линзы, К1 - К10 - дипольные корректора, М1 - M4 сцинтилляционные мониторы, ВРМ1 - ВРМ3 - датчики положения центра тяжести пучка, IP - точка инжекции, СР - точка взаимодействия электронного и лазерного пучков (место расположения оптического резонатора).

Периметр накопителя составляет 15,418 м. Поворотные магниты (B1 - B4) имеют радиус поворота 0,5 м, угол поворота $90^{\circ}$, магнитное поле на максимальной рабочей энергии электронного пучка (200 МэВ) составляет 
1,3 Тл. Промежуток инжекции (B1 - В2) имеет ненулевую дисперсию $\mathrm{D}_{\mathrm{x}} \neq 0$. Промежуток взаимодействия (B3 $\mathrm{B} 4)$, содержащий оптический резонатор - бездисперсионный $\left(\mathrm{D}_{\mathrm{x}}=0\right)$. Так как нет поворотов в вертикальной плоскости, вертикальная дисперсия всюду в накопителе равна нулю $\left(\mathrm{D}_{\mathrm{z}}=0\right)$. Для фокусировки электронного пучка в точке взаимодействия используются четыре квадрупольных линзы (Q1 - Q4, Q17 - Q20). Такая схема фокусировки дает возможность получить минимальные значения горизонтальной $(0,23$ м) и вертикальной $(0,21$ м) амплитудных функций в точке взаимодействия. Дисперсионные и функции фокусировки накопительного кольца «НЕСТОР» [9] показаны на рис.2 а,б.

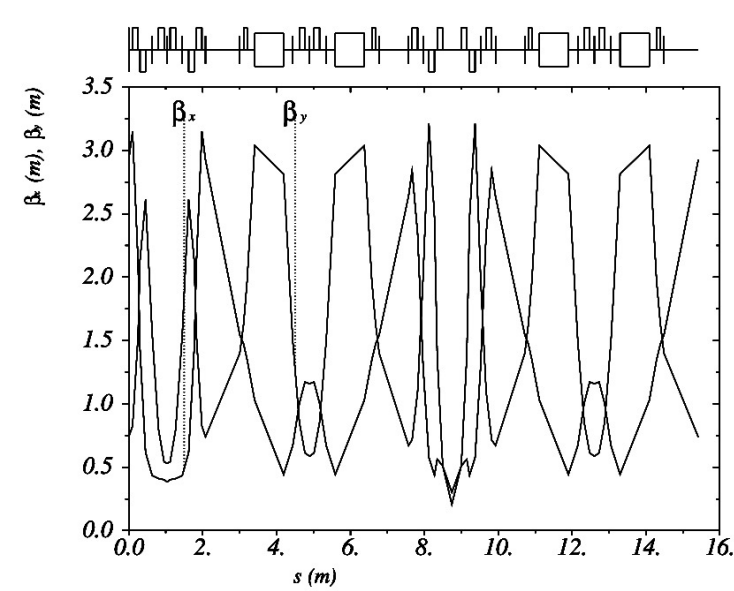

a)

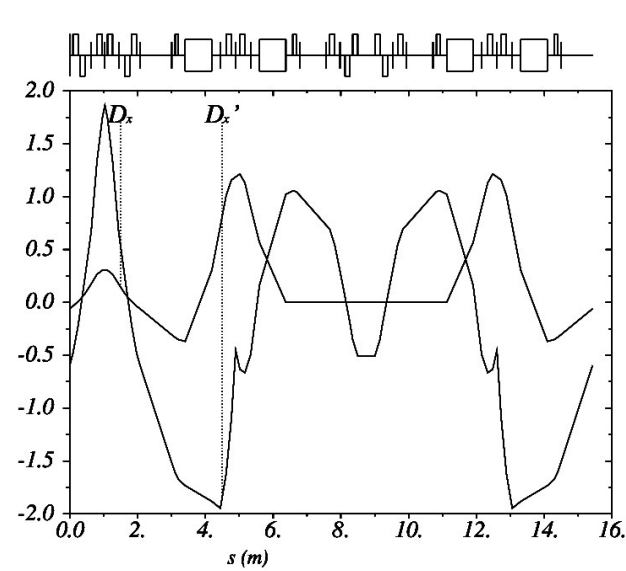

б)

Рис.2. Функции фокусировки накопительного кольца «НЕСТОР»

a) амплитудные функции, б) дисперсионные функции $\left(\mathrm{D}_{\mathrm{x}}, \mathrm{D}_{\mathrm{x}}^{\prime}\right)$

\section{ОШИБКИ ЮСТИРОВКИ ЭЛЕМЕНТОВ МАГНИТНОЙ СИСТЕМЫ}

Моделирование динамики электронного пучка проводилось с помощью программных пакетов MAD-X [10] и DeCA [11]. При моделировании влияния ошибок юстировки магнитных элементов на динамику электронного пучка задавались ошибки юстировки для 4 поворотных магнитов (B1 - B4) и для 20 квадрупольных линз (Q1 Q20). В накопителе используется два вида квадрупольных линз: 16 квадрупольных линз с эффективной длинной 0,15 м и 4 линзы с эффективной длиной 0,1 м.

Задаваемые ошибки юстировки различны для поворотных магнитов и для разных типов квадрупольных линз. В таблицах 1-3 приведены ошибки юстировки магнитных элементов, которые использовались при моделировании (где dx - сдвиг в горизонтальной плоскости, dz - в вертикальной, ds - сдвиг по азимуту, хs, zs, $\mathrm{xz}$ - повороты в соответствующих плоскостях). Эти ошибки юстировки получены из оценки точности используемой методики выстановки магнитных элементов [12].

Таблица 1

Ошибки юстировки для 16 квадрупольных линз с эффективной длиной 0,15 м.

\begin{tabular}{|c|c|c|c|}
\hline Сдвиг в плоскости & Rms (мкм) & Угловой сдвиг & Rms (мрад) \\
\hline $\mathrm{dx}$ & 100 & $\mathrm{xs}$ & 0,7 \\
\hline $\mathrm{dz}$ & 100 & $\mathrm{zs}$ & 0,7 \\
\hline $\mathrm{ds}$ & 300 & $\mathrm{xz}$ & 1,25 \\
\hline
\end{tabular}

Таблица 2

Ошибки юстировки для 4 квадрупольных линз с эффективной длиной 0,1 м.

\begin{tabular}{|c|c|c|c|}
\hline Сдвиг в плоскости & Rms (мкм) & Угловой сдвиг & Rms (мрад) \\
\hline $\mathrm{dx}$ & 100 & $\mathrm{xs}$ & 1 \\
\hline $\mathrm{dz}$ & 100 & $\mathrm{zs}$ & 1 \\
\hline $\mathrm{ds}$ & 300 & $\mathrm{xz}$ & 1,25 \\
\hline
\end{tabular}

Таблица 3

Ошибки юстировки для 4 поворотных магнитов.

\begin{tabular}{|c|c|c|c|}
\hline Сдвиг в плоскости & Rms (мкм) & Угловой сдвиг & Rms (мрад) \\
\hline $\mathrm{dx}$ & 100 & $\mathrm{xs}$ & 0,33 \\
\hline $\mathrm{dz}$ & 100 & $\mathrm{zs}$ & 0,33 \\
\hline $\mathrm{ds}$ & 300 & $\mathrm{xz}$ & 0,20 \\
\hline
\end{tabular}


Эти ошибки юстировки приводят к следующим среднеквадратичным отклонениям равновесной орбиты относительно идеальной (рис.3 а, б).

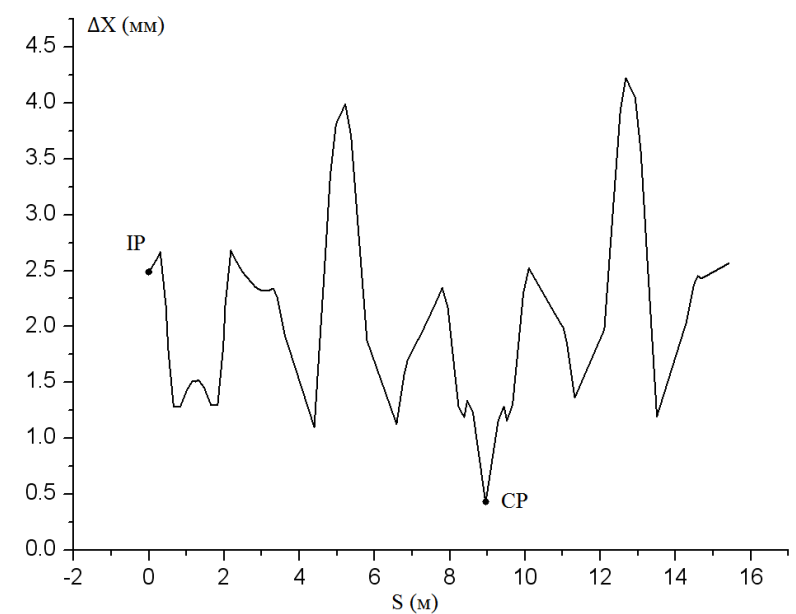

a)

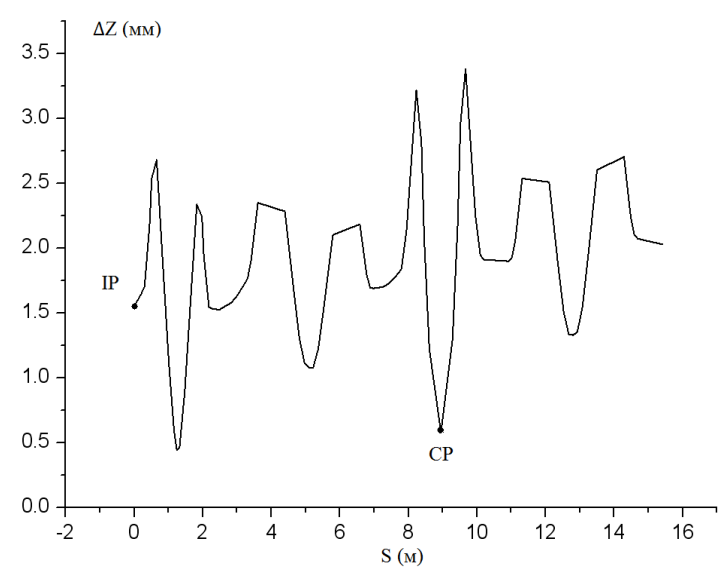

б)

Рис.3. Среднеквадратичные отклонения равновесной орбиты пучка относительно идеальной IP - точка инжекции, СР - точка взаимодействия

а) горизонтальная плоскость, б) вертикальная плоскость

Как можно видеть из рис. 3 a, б, среднеквадратичные отклонения положения равновесной орбиты в точке взаимодействия (СР) достигают 0,4 мм в вертикальной плоскости и 0,5 мм в горизонтальной. Среднеквадратичные поперечные размеры электронного и лазерного сгустков в этой точке составляют всего несколько десятков микрометров. Поэтому, такие смещения положения электронного пучка могут сильно осложнить настройку работы источника жесткого излучения.

Из рис. 3 видно, что в точке инжекции отклонения положения равновесной орбиты составляют 1,6 мм в вертикальной плоскости и 2,6 мм в горизонтальной. В то же время, физическая апертура ускорителя в точке инжекции составляет всего \pm 4 мм в вертикальной плоскости и \pm 20 мм в горизонтальной, что связано с конструкционными особенностями импульсного магнита системы инжекции (инфлектор). Из-за малости физической апертуры инфлектора до 65\% инжектируемых частиц теряется на этом азимуте.

Кроме того, два технологических ограничения в точках установки датчиков положения пучка (BMP1 и BMP2) уменьшают эффективную физическую апертуру накопителя [14]. На этих апертурах теряется до $15 \%$ инжектируемых частиц. На рис. 4 показаны физические апертуры в местах установки датчиков положения пучка.

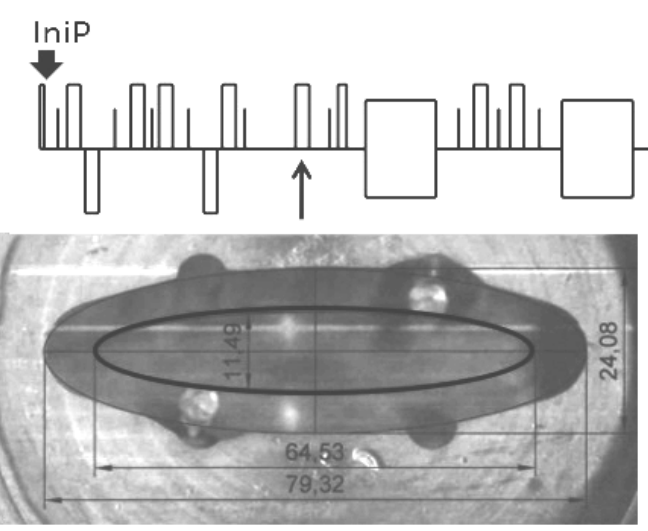

Эффективная апертура 32,3×5,75 мм

a)

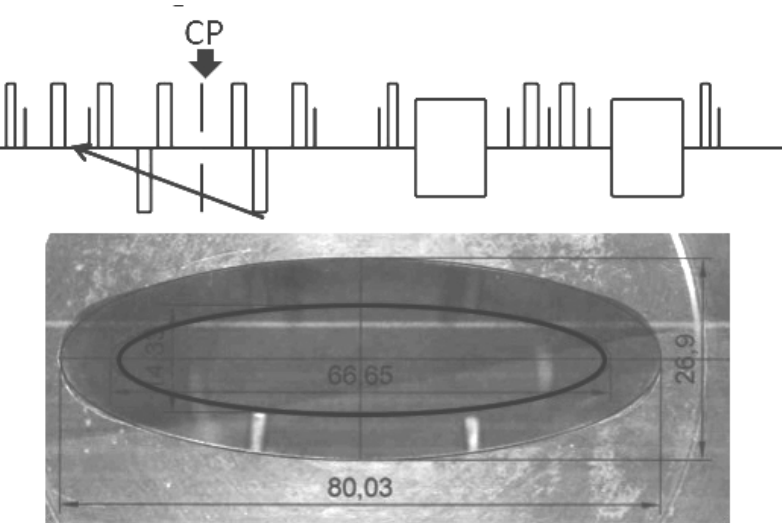

Эффективная апертура 33,3×7,16 мм

б)

Рис.4. Физические апертуры в местах установки датчиков положения пучка a) монитор положения пучка BMP1, б) монитор положения пучка BMP2

\section{ОЦЕНКИ ЭФФЕКТИВНОСТИ ИНЖЕКЦИИ}

Моделирование показало, что в идеальных условиях (нет смещений равновесной орбиты из-за ошибок юстировки) в кольце накапливается $85 \%$ инжектируемых частиц, остальные теряются на физических апертурах накопителя. Потери частиц связаны с тем, что параметры Твисса (они определяют ориентацию эллипса на фазовой плоскости колебаний циклической структуры) на выходе канала транспортировки не согласованы с 
параметрами Твисса в точке инжекции в накопитель [13].

Частота ускоряющего ВЧ напряжения в накопительном кольце составляет 700 МГц, а в линейном ускорителе 2800 МГц. Поэтому, при инжекции будет захватываться только каждый четвертый электронный сгусток, а три других будут теряться на физических апертурах накопителя.

Эффективность транспортировки электронного пучка через канал инжекции составляет около $70 \%$, так как между поворотными магнитами инжекционного канала размещен коллиматор для уменьшения энергетического разброса в пучке.

Если учесть все вышесказанное и взять за $100 \%$ количество электронов на выходе линейного ускорителя, то в идеальных условиях в кольце будет накапливаться около $15 \%$ частиц. Для накопления проектного тока (36 сгустков по $10 \mathrm{MA}$ ) при частоте инжекции 1 Гц и импульсном токе линейного ускорителя, равном $100 \mathrm{MA}$, потребуется 24 секунды.

Моделирования показало, что в $50 \%$ случаев при используемой системе юстировки магнитных элементов можно добиться накопления электронного пучка без коррекции равновесной орбиты. В случае если накопления не происходит, необходимы алгоритмы проводки первого оборота.

Для успешной коррекции равновесной орбиты на каждое бетатронное колебание в соответствующей плоскости должно приходиться четыре датчика положения пучка [14]. Частоты бетатронных колебаний накопителя «НЕСТОР» равны 3,13 в горизонтальной плоскости и 1,78 в вертикальной. Другими словами, для качественной коррекции равновесной орбиты необходимо 12 датчиков в горизонтальной плоскости и 7 в вертикальной.

\section{ВЫВОДЫ}

Моделирование показало, что в $50 \%$ случаев при используемой системе юстировки магнитных элементов можно добиться накопления электронного пучка без использования каких-либо дополнительных алгоритмов. В случае если накопления не происходит, необходимо использовать специальные алгоритмы проводки первого оборота и накопления. Оценки показывают, что для качественной коррекции равновесной орбиты необходимо иметь 12 датчиков положения пучка в горизонтальной плоскости и 7 в вертикальной.

\section{СПИСОК ЛИТЕРАТУРЫ}

1. Motz H. Applications of the radiation from fast electron beams // Journal of Apply Physics. - 1951. -Vol. 22. - No. 527. P. 527-531.

2. Landecker K. Possibility of frequency multiplication and wave amplification by means of some relativistic effects // Physical Review. - 1952. - Vol. 86. - No.6. - P. 852-855.

3. Artumanyan F., Tumanyan V. Compton effect on relativistic electrons and the possibility of generation of hard radiation // JETP. - 1963. - Vol. 44. - P. 2101.

4. Milburn R. // Thomson Scattering of Optical Radiation from an Electron Beam // Physical Review Letters. - 1963. - Vol. 10. P. 75 .

5. Gladkikh P., Karnaukhov I. et. al. Physical Grounding H-100M // Internal report of NSC KIPT. - 1998. - P. 7-30.

6. Telnov V. Principles of photon colliders // Nuclear Instruments and Methods in Physics Research A. - 1995. - Vol. 355. P. 3-18.

7. Zelinsky A., Shcherbakov A. et al. The Kharkov X-ray generator facility NESTOR // Proceedings of IPAC. - 2013. P. 2253-2255.

8. Gladkikh P., Karnaukhov I. et al. The start of X-ray generator NESTOR commissioning // VANT. -2013. - Vol. 6(88). P. 20-23.

9. Zelinsky A., Kalamaiko A. et al. The First Results of the NESTOR Commissioning NESTOR // Proceedings of IPAC. - 2013. P. 225-227.

10. Iselin F.C. The MAD Program. Physical Methods Manual // CERN/SL. -1992

11. Gladkikh P., Strelkov M., Zelinsky A. The Application Package DECA for Calculating Cyclic Accelerators // Proceedings of PAC-93. - 1993. - P. 194-196.

12. Drebot I., Karnaukhov I., ea at. Survey and alignment strategy for compton x-ray generator NESTOR // Proceedings of IPAC2010. - 2010. - P. 4053-4055.

13. Zelinsky A., Kalamaiko A. et al. Simulation of the beam dynamics due to alignment error of magnetic elements in the Nestor ring // Proceedings of XIV conference on high energy physics, nuclear physics and accelerators. - 2016. - P. 62.

14. Dinev D. Closed orbit correction in synchrotrons // Physics of elementary particles and nuclear physics. - 1997. - Vol. 4(28). P. 1013-1060. 